\title{
Zu den Vorschlägen der Gesundheitsdirektorenkonferenz
}

Vor rund zwei Wochen veröffentlichte die GDK (Schweizerische Konferenz der kantonalen Gesundheitsdirektorinnen und -direktoren) eine sehr interessante Mitteilung zur Gesundheitspolitik im allgemeinen sowie zum Ende des Zulassungsstopps und zu den Versorgungssystemen («Managed Care») im besonderen.

Interessant ist dieses Dokument vor allem deshalb, weil es wenige Tage vor dem Beginn der neuen Legislatur aufzeigt, dass die Positionen der Kantone und die Positionen, die wir bereits seit mehreren Jahren vertreten, in einigen wesentlichen Punkten übereinstimmen!

Befassen wir uns also mit diesen Übereinstimmungen ... und auch mit den unterschiedlichen Auffassungen:

- Zunächst ist festzuhalten, dass die GDK erkannt hat, dass Managed Care ohne starke finanzielle Anreize für die Patientinnen und Patienten nur ein frommer Wunsch ist. Wir weisen seit langem auf diese Tatsache hin: Wenn sich dieses System entwickeln soll, müssen entsprechende Anreize geschaffen werden.

Wir hoffen daher sehr, dass auch die Gesundheitskommission des Nationalrats diesen wichtigen Punkt anerkennen wird, wenn sie sich dann endlich mit diesem Dossier befassen wird.

- Die GDK hält richtigerweise fest, dass ein besserer Risikoausgleich und eine Qualitätssicherung erforderlich sind.

- Wir sind hingegen nicht der Auffassung, dass eine gemeinsame Budgetverantwortung in den ManagedCare-Netzwerken für obligatorisch erklärt werden sollte.

Unserer Meinung nach gibt es überhaupt keinen Grund, hinsichtlich dieses Punkts jedes Detail vorzuschreiben. Da sich Managed Care nur auf der Grundlage von bilateralen Verträgen zwischen Ärzten und Versicherern entwickeln kann, ist es unerlässlich, dass diesen Partnern Vertrauen geschenkt wird und dass sie sich frei absprechen können. Auf diese Weise kann der gesamten Vielfalt der Bedürfnisse Rechnung getragen werden.

Wir unterstützen Managed Care als flexible und angemessene Lösung für die Probleme des Gesundheitssystems. Ebenso sind wir aber überzeugt, dass die angestrebten Lösungen durch Zwangsmassnahmen und systematische Verpflichtungen unnötigerweise beeinträchtigt werden.
- Die Auffassung der GDK zum Ende des Zulassungsstopps ist ebenfalls interessant, aber weniger begeisternd!

Was die Grundversorger anbelangt, schlägt die GDK vor, diese Massnahme aufzuheben - was durchaus zu begrüssen ist. Doch für die Spezialisten will sie den Zulassungsstopp in einer «verbesserten» Form - die noch festgelegt werden müsste - beibehalten.

Die FMH hat zwar bereits vor einigen Monaten festgehalten, dass nach dem 3. Juli 2008 eine gewisse Regulierung möglich sein sollte, doch mit einer so schematischen Vorstellung lassen sich keine Probleme lösen. Wenn auf eine solche Weise ein Keil zwischen die Ärzteschaft getrieben wird, können weder für die Probleme der Randregionen noch für die Probleme im Zusammenhang mit der Ärztedemographie im allgemeinen realisierbare Lösungen gefunden werden. Sogar die Grundversorger teilen diese Ansicht!

Aus den Erfahrungen der letzten Jahre geht klar hervor, dass die Ärztedemographie nur auf der Ebene der Kantone richtig geregelt werden kann. Diese müssen über einen ausreichenden Spielraum verfügen, um ihrer eigenen Realität und ihren Bedürfnissen Rechnung tragen zu können. Dank der Vertrautheit mit den lokalen Verhältnissen können sie auch Massnahmen vorschlagen, mit denen die Ärzte in Ausbildung berücksichtigt werden - das ist wohl das mindeste!

So bestehen also einige Übereinstimmungen und selbstverständlich auch gewisse unterschiedliche Auffassungen. Die letzteren sind von erheblicher Bedeutung und rechtfertigen entschlossene politische Aktivitäten unsererseits. Trotz teilweise unterschiedlicher Positionen ist eine Zusammenarbeit im Bereich dieses Dossiers denkbar und anzustreben.

Sehr bemerkenswert ist auch folgendes: Mit unseren politischen Zielsetzungen, die sowohl differenziert und präzise als auch offen und klar festgelegt sein sollen, lassen sich nun übereinstimmende Standpunkte finden, obwohl sie manchmal als unrealistisch abqualifiziert wurden. Dies zeigt uns, dass wir auf dem richtigen Weg sind!

Die Zusammenarbeit mit der GDK ist bekanntlich nicht neu - das jüngste Beispiel war die Frage der ärztlichen Grundversorgung. Doch es ist erfreulich und durchaus wünschenswert, dass sich diese Zusammenarbeit in den kommenden Jahren noch weiterentwickeln kann.

Dr. med. Jacques de Haller, Präsident der FMH 